\title{
Genome-wide approaches in the study of common fig in the Nikita Botanical Gardens
}

\author{
Mitrofanova I.*, Krivenko O., Kuleshova O., Emirsaliev A., Tsyupka V. \\ Federal State Funded Institution of Science "The Nikita Botanical Gardens - National Scientific Center \\ of the RAS" (NBG-NSC), Yalta, Russia \\ * email: invitro_plant@mail.ru
}

Fig is one of the most ancient subtropical fruit crops using in food, cosmetic, and soap industries as well as in medicine, which industrial production is hindered by the lack of intensive cultivation technologies. Genome-wide approaches provide an effective tool for the development and implementation of the modern molecular breeding strategies in the fig cultivation. The Nikita Botanical Gardens (NBG-NSC) has a unique common fig collection represented by 267 cultivars and hybrid forms including of local breeding. A comprehensive genomic study of the common fig collection started in the NBG-NSC a few years ago and four main researcher projects are ongoing. First version of the reference genome of the local cultivar Ficus carica 'Sabrutsiya Rozovaya' was obtained by hybrid assembly using combined long and short read genome wide sequencing datasets. To identify the molecular determinants of fig resistance to complex abiotic stress, we investigate changes in a differential gene expression during adaptation of in vitro cultivate plants to in vivo and ex situ conditions based on the transcriptomes analysis of the leaves obtained from three $F$. carica cultivars with alternative phenotypes. Methodological framework for the investigation of the molecular mechanisms of cell differentiation in plants based on the single-cell RNA-sequencing analysis are developing using in vitro $F$. carica culture. A detail description of the genetic diversity of the fig collection, including sequencing of the key cultivars, genotyping of the collection, with a focus on the traits responsible for resistance to abiotic and biotic stress, will make a base for the development of advanced genomic breeding and cultivation fig technologies in the NBG-NSC.

Acknowledgements: The first two projects are supported by the grant No. 075-15-20191670 of the Ministry of Science and Higher Education of Russian Federation and done in Kurchatov Genomic Center - NBG-NSC; the other are conducted in the frame of the State Task No. 0829-2019-0038 of FSFIS “NBG-NSC”. 